\title{
Diffusion tensor imaging of the cortical plate and subplate in very-low-birth-weight infants
}

\author{
Jeroen Dudink • Jan Buijs • Paul Govaert • \\ Arjen L. van Zwol • Nikk Conneman • \\ Johannes B. van Goudoever • Maarten Lequin
}

Received: 9 September 2009 /Revised: 2 December 2009 / Accepted: 24 January 2010 /Published online: 27 March 2010

(C) The Author(s) 2010. This article is published with open access at Springerlink.com

\begin{abstract}
Background Many intervention studies in preterm infants aim to improve neurodevelopmental outcome, but short-term proxy outcome measurements are lacking. Cortical plate and subplate development could be such a marker.

Objective Our aim was to provide normal DTI reference values for the cortical plate and subplate of preterm infants. Materials and methods As part of an ongoing study we analysed diffusion tensor imaging (DTI) images of 19 preterm infants without evidence of injury on conventional MRI, with normal outcome (Bayley-II assessed at age 2), and scanned in the first 4 days of life. Fractional anisotropy (FA) and apparent diffusion coefficient (ADC) values in the frontal and temporal subplate and cortical plate were
\end{abstract}

J. Dudink $(\bowtie) \cdot$ P. Govaert • A. L. van Zwol • N. Conneman •

J. B. van Goudoever

Division of Neonatology, Department of Paediatrics,

Erasmus MC-Sophia Children's Hospital,

Rotterdam, The Netherlands

e-mail: j.dudink@erasmusmc.nl

\section{Lequin}

Division of Paediatrics, Department of Radiology, Erasmus MC-Sophia Children's Hospital,

Rotterdam, Zuid-holland 3015 GJ, The Netherlands

\section{J. Buijs}

Division of Neonatology, Department of Paediatrics,

Máxima Medical Center, Veldhoven, The Netherlands

Present Address:

J. Dudink

Division of Paediatrics, Department of Radiology,

Erasmus MC-Sophia Children's Hospital,

Dr. Molenwaterplein 60, Rotterdam,

Zuid-holland 3015 GJ, The Netherlands measured in single and multiple voxel regions of interest (ROI) placed on predefined regions.

Results Using single-voxel ROIs, statistically significant inverse correlation was found between gestational age (GA) and FA of the frontal $(r=-0.5938, P=0.0058)$ and temporal $(r=-0.4912, P=0.0327)$ cortical plate. ADC values had a significant positive correlation with GA in the frontal $(r=$ $0.5427, P=0.0164)$ and temporal $(r=0.5540, P=0.0138)$ subplate.

Conclusion Diffusion tensor imaging allows in vivo exploration of the evolving cortical plate and subplate. We provide FA and ADC values of the subplate and cortical plate in very-low-birth-weight (VLBW) infants with normal developmental outcome that can be used as reference values.

Keywords Cortical plate $\cdot$ Subplate $\cdot$ Diffusion tensor imaging · Fractional anisotropy $\cdot$ Apparent diffusion coefficient . Very low birth weight · Infant · Preterms

\section{Introduction}

Advances in neonatal intensive care have led to significant improvement in survival of very-low-birth-weight (VLBW) infants $[1,2]$. However survivors of preterm birth still show a high incidence of neurodevelopmental disability. Up to $25-50 \%$ of VLBW infants have cognitive and attention deficits, often without marked motor deficits [3-5]. Early detection of abnormal brain maturation is important in the design of preventive, protective and rehabilitative strategies for the management of VLBW infants. White matter injury is believed to be the most common type of injury seen in preterm infants and is associated with neurological deficits seen in VLBW infants later in life [6,7]. However, it is difficult to relate cognitive and attention deficits entirely to 
white matter disease. Instead it seems more likely that these deficits are secondary to sensory deafferentation and/or axotomy [8].

The human cerebral cortex develops through a series of stages during which complex histogenetic events occur in several transient anatomic layers. Many of these layers disappear as the fetus approaches term. The laminar structure of the developing cortex comprises the marginal zone, cortical plate (CP) and the subplate (a band of cells lying below the developing cortical plate).

The subplate is a transient laminar compartment of the human fetal cerebral wall that plays an important role in the developing cortical architecture. The subplate develops around week 13 of gestation and gradually disappears at 32-34 weeks. The structure is thought to be the main synaptic zone of the human fetal cortex based on the rich input of 'waiting' afferents from cortical plate and thalamus. It plays a major role during the phase of cortical target area selection [9]. The subplate is a site of early neural activity, it is seen as substrate for functional plasticity and is considered to be selectively vulnerable for hypoxic/ ischaemic injury. All this renders the subplate a structure of great interest in the study of preterm brain injury [9-12].

The subplate can be visualized with conventional and diffusion tensor magnetic resonance imaging (DTI) [13-16]. In contrast to conventional anatomical imaging, DTI can provide information about the microstructural organization within neural tissue in vivo [17]. Studies have shown that diffusion anisotropy can be used to study brain development $[15,18]$. DTI can thus be used to quantify microstructural organization of the subplate in vivo. Maas et al. [14] used DTI to delineate early laminar organization of the cerebrum in two extremely premature infants. Gupta et al. [15] performed DTI on 15 aborted fetuses and 5 term infants to look at regional FA values including the subplate. There is a lack of DTI reference values for the subplate in healthy VLBW infants in the first week of life. Reference values of the subplate could be used as a tool to quantify the efficacy of interventions in clinical trials. In addition, these reference values might enable the paediatrician to identify infants at risk for suboptimal development already in the first phase of life.

We set up a prospective study aimed at providing DTI reference values for the CP and subplate zone in VLBW infants.

\section{Materials and methods}

\section{Patients}

The Erasmus MC Ethical Review Board approved the study and written informed parental consent was obtained for each patient. The MR scans used in this study had been obtained within the framework of a study in which premature infants of different gestational ages underwent serial conventional and DTI acquisitions to evaluate white matter development. All MR scans were obtained without sedation. The inclusion criteria for our study were birth at gestational age (GA) of 25-32 weeks, no evidence of cerebral injury on conventional MRI (T1- and T2-weighted images) and scanned within 4 days of birth. Developmental outcome assessed at age of 2 years needed to be normal. Gestational age was calculated from the mother's last menstrual period or estimated from early US $(<18$ weeks of pregnancy). Exclusion criteria were ventriculomegaly, congenital infection, brain malformation or a multiple congenital anomaly syndrome. During the 25 months of the study period (March 2004 to April 2006), 41 infants were scanned and 32 met our study criteria. Four MR examinations were excluded due to severe motion degradation and 9 due to other artefacts, so 19 infants were included.

\section{Neurodevelopment monitoring}

All children were neurologically assessed at 2 years of age and Bayley scales of infant development second edition (BSIDII) were used to monitor neurodevelopment [19]. All children had shown normal neurodevelopment according to their neurological examination and Bayley scores [Bayley scores within one standard deviation (SD) of the mean were considered within normal limits, i.e. $>85$ ].

\section{Conventional MR imaging}

Images were acquired using standard scanning protocols. All imaging was performed on a 1.5-T GE EchoSpeed scanner (GE Medical Systems, Milwaukee, WI, USA). The imaging protocol included T1-W (spin-echo, TR $500 \mathrm{~ms}$, TE $11 \mathrm{~ms}$ ) and T2-W sequences (spin-echo, TR 3,000 ms, TE $120 \mathrm{~ms}$ ) with a slice thickness of $4 \mathrm{~mm}$ and $0.4-\mathrm{mm}$ gap. For DTI an echo planar sequence with diffusion gradients applied in 25 non-collinear directions was used with a slice thickness of $3 \mathrm{~mm}$ and no gap $\left(b=1,000 \mathrm{~s} / \mathrm{mm}^{2}\right)$. An average of 20 slices was recorded within 4 min using TR 9,150 ms and TE 98-91 ms. The field of view (FOV) was $20 \mathrm{~cm}$, the scan matrix $128 \times 128$ and the reconstruction matrix $256 \times 256$. All children were scanned using an MRcompatible incubator with a specialized high-sensitivity neonatal head coil (Lammers Medical Technology, Lubeck, Germany). Pads around the infant's head kept movement to a minimum. Mouldable earplugs and neonatal earmuffs were used to reduce noise. The incubator provided controlled temperature and humidity as well as MR compatible pulse oximetry and ventilation. 


\section{Diffusion tensor post-processing}

Diffusion tensor images were transferred to a GE Advantage Windows workstation (General Electric Medical Systems, Milwaukee, WI, USA) for post-processing using Functool software (General Electrics).

DTI measures the diffusion of water in each voxel and the extent to which water diffuses in particular directions as a result of the microstructural characteristics of the tissue imaged. FA measures the fraction of the magnitude of the diffusion tensor that can be ascribed to anisotropic diffusion [20-22]. For isotropic diffusion $(\lambda 1=\lambda 2=\lambda 3), F A$ is zero, and in the case where there is a strongly preferred direction of diffusion $(\lambda 1>>\lambda 2=\lambda 3)$, FA approaches a value of one. The apparent diffusion coefficient (ADC) was calculated from the eigenvalues for each voxel as follows: $(\lambda 1+\lambda 2+$ $\lambda 3) / 3$, and provides the overall magnitude of water diffusion. $\mathrm{ADC}$ is independent of anisotropy and is also a useful parameter for brain maturation [23].

Two strategies for regions of interest (ROI) placement were used. First we placed two rows (right and left hemisphere) of single-voxel ROIs along a straight line on an axial slice through the foramen of Monro (Fig. 1); this line extended along the long axis of the lateral ventricle through the subplate and CP. Next we placed two rows of single-voxel ROIs on an axial slice through the pedunculi of the brainstem from the top of the temporal horn to the right and left CPs (Fig. 1). We identified the voxel most representative of the $\mathrm{CP}$ and subplate visually on the b0-reference image. We cross correlated this with the T1- and T2-W scans and the ADC and FA maps.

In the second method we placed standard-size, round 16-voxel ROIs in the region of the subplate on the same axial slices as mentioned in the first method. In the temporal regions it was more difficult to place the 16-voxel ROI in the centre of the $\mathrm{CP}$ and we decided to use this method for the subplate only because we suspected too much interference from partialvolume effects. We identified the subplate visually on the b0reference image. We cross correlated this with the T1- and T2$\mathrm{W}$ scans and the ADC and FA maps (Fig. 2). Two researchers experienced in neonatal DTI achieved consensus on ROI placement and measurement readings. For every ROI, $\lambda 1, \lambda$ 2, $\lambda 3$ were measured and FA and ADC were calculated.
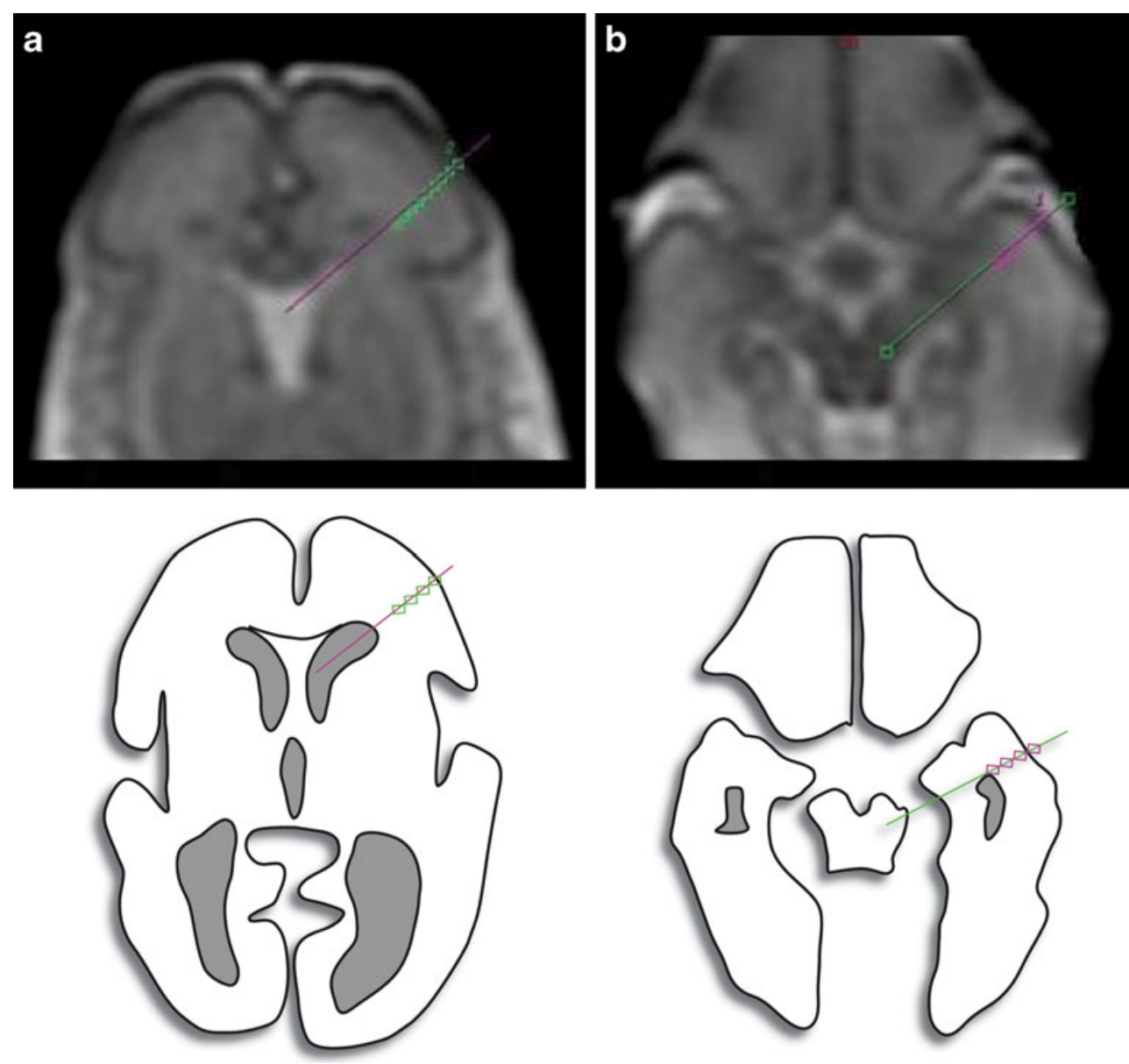

Fig. 1 Top image shows ROI placement (row of single voxels) in both frontal (a) and temporal (b) regions. The image below is a schematic diagram that shows the same ROI placement 

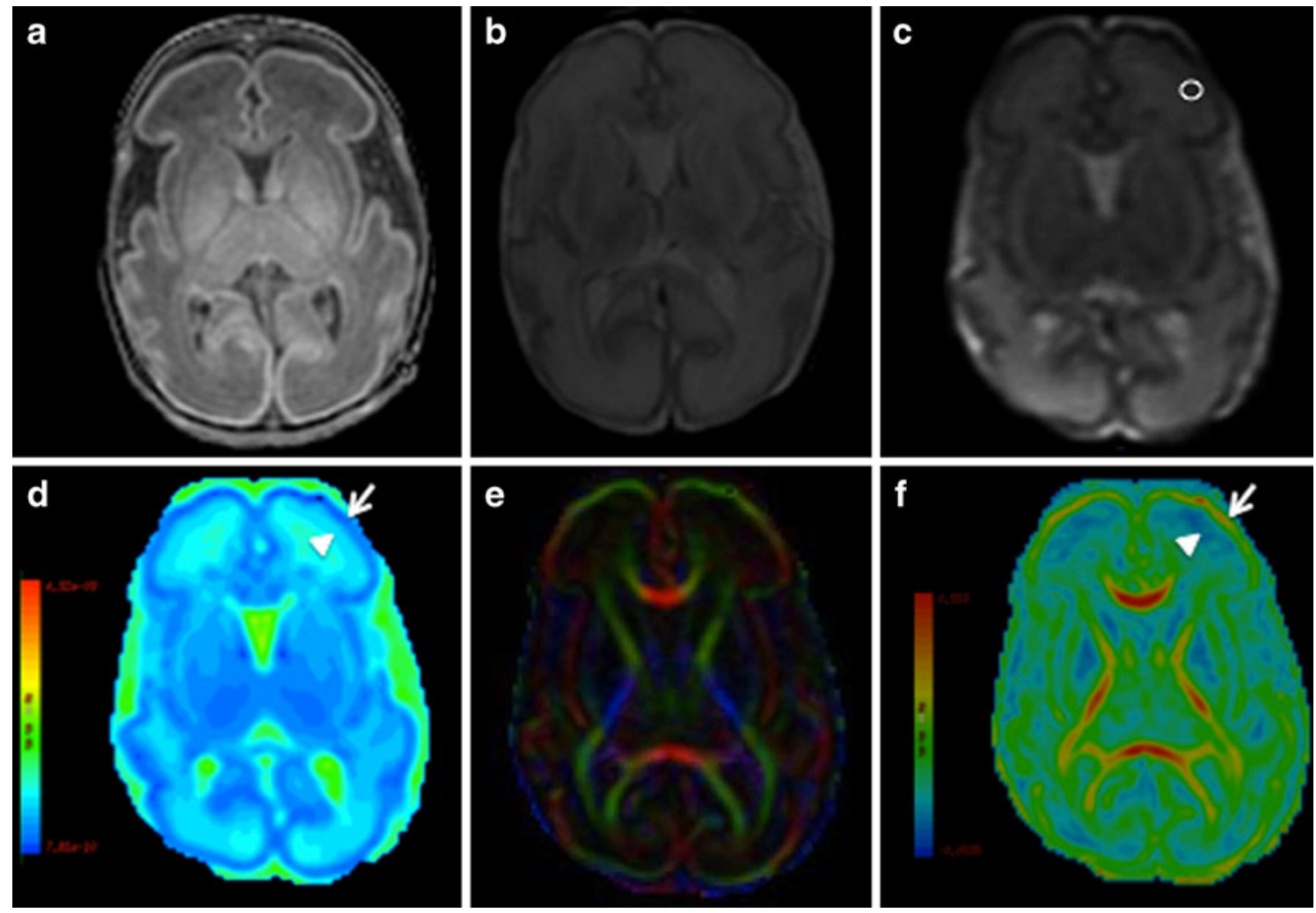

Fig. 2 Different MRI scan sequences of the same infant at 30 weeks GA. T1-W (a), T2-W image (b) and b0 image (c) show low signal intensity (SI) of the CP and a ROI placed in area of the subplate. ADC map (d) shows low (dark blue) ADC values of the cortex (arrow) and intermediate ADC values of the subplate (arrowhead). Colour map (e)

\section{Statistical analysis}

The relationship between FA and ADC of the CP and GA and between the subplate and GA was analyzed by correlation analysis (Pearson product moment correlation, SPSS 13.0.1, SSPS Inc., Chicago, IL).

\section{Results}

\section{Patient characteristics}

Gestational age at birth ranged from 26 to 31 weeks (mean $29^{+1}$ weeks). All infants were appropriate for GA. Mean birth weight was $1,135 \mathrm{~g}$ and mean head circumference was $26 \mathrm{~cm}, 9$ of the infants were girls. In 91\% antenatal steroids were given. The median Apgar score at 5 min was 9 (range 5-10). No infections were reported in the first week of life. Apart from one, all infants received respiratory support in the first week of life.

\section{FA and ADC calculations}

For both ROI methods, we plotted the calculated FA and ADC values of the $\mathrm{CP}$ and the subplate against gestational age (GA). (red representing right-left, green representing anteroposterior and blue representing superoinferior anatomical directions) and FA map (f), show high FA values of the cortex (arrow) and low FA values (blue) of the subplate zone (arrowhead)

No statistically significant differences based on a two-tailed paired Student's $t$ test (at a significance level of $P<0.05$ ) were found comparing FA and $\mathrm{ADC}$ values between the left and right hemispheres in each subject. Therefore, the values obtained from the left and right were averaged to obtain the mean FA and ADC of the different ROIs (Table 1).

\section{FA and ADC of single-voxel ROIs}

A statistically significant inverse correlation was found between GA and FA of the frontal $(r=-0.5938, P=0.0058)$ and temporal $(r=-0.4912, P=0.0327) \mathrm{CP}$ (Figs. 3 and 4$)$. No significant correlation between $\mathrm{ADC}$ and the $\mathrm{CP}$ were detected. ADC had a significant correlation with GA in the frontal $(r=0.5427, P=0.0164)$ and temporal $(r=0.5540, P=$ 0.0138 ) subplate (Fig. 3). No correlation was found between FA values in the frontal and temporal subplate and GA.

FA and ADC of ROIs of standard 16-pixel size

A statistically significant correlation was found between GA and $\mathrm{ADC}$ of the frontal $(r=0.505, P=0.027)$ and temporal $(r=0.301$, $P=0.015$ ) subplate (Fig. 4). No significant correlations were found between FA of the frontal and temporal subplate and GA. 
Table 1 Mean FA and ADC values in the frontal and temporal subplate

\begin{tabular}{|c|c|c|c|c|}
\hline & \multicolumn{2}{|c|}{ Frontal subplate mean (SD) } & \multicolumn{2}{|c|}{ Temporal subplate mean (SD) } \\
\hline & Right & Left & Right & Left \\
\hline \multicolumn{5}{|l|}{ Single-voxel ROI } \\
\hline FA & $0.069(0.02)$ & $0.061(0.02)$ & $0.08(0.02)$ & $0.075(0.02)$ \\
\hline $\mathrm{ADC} \times 10^{-3} \mathrm{~mm}^{2} / \mathrm{s}$ & $1.539(0.14)$ & $1.519(0.13)$ & $1.593(0.14)$ & $1.609(0.16)$ \\
\hline \multicolumn{5}{|l|}{ 16-voxel ROI } \\
\hline FA & $0.09(0.03)$ & $0.08(0.03)$ & $0.103(0.02)$ & $0.09(0.02)$ \\
\hline $\mathrm{ADC} \times 10^{-3} \mathrm{~mm}^{2} / \mathrm{s}$ & $1.509(0.16)$ & $1.52(0.17)$ & $1.509(0.09)$ & $1.527(0.12)$ \\
\hline
\end{tabular}

\section{Discussion}

We provide reference values for the evaluation of DTI images of the CP and subplate in VLBW infants with normal outcome at 2 years of age. A statistically significant inverse correlation was measured between GA and FA values of the frontal and temporal cortical $\mathrm{CP}$. ADC values were found to have a significant positive correlation with GA in the frontal and temporal subplate.

$\mathrm{ADC}$ and FA values of cortical plate

Our observation of decreased cortical FA values with increasing GA after 26 weeks seems consistent with published data $[15,18,24]$. Before 26 weeks a gradual increase in the
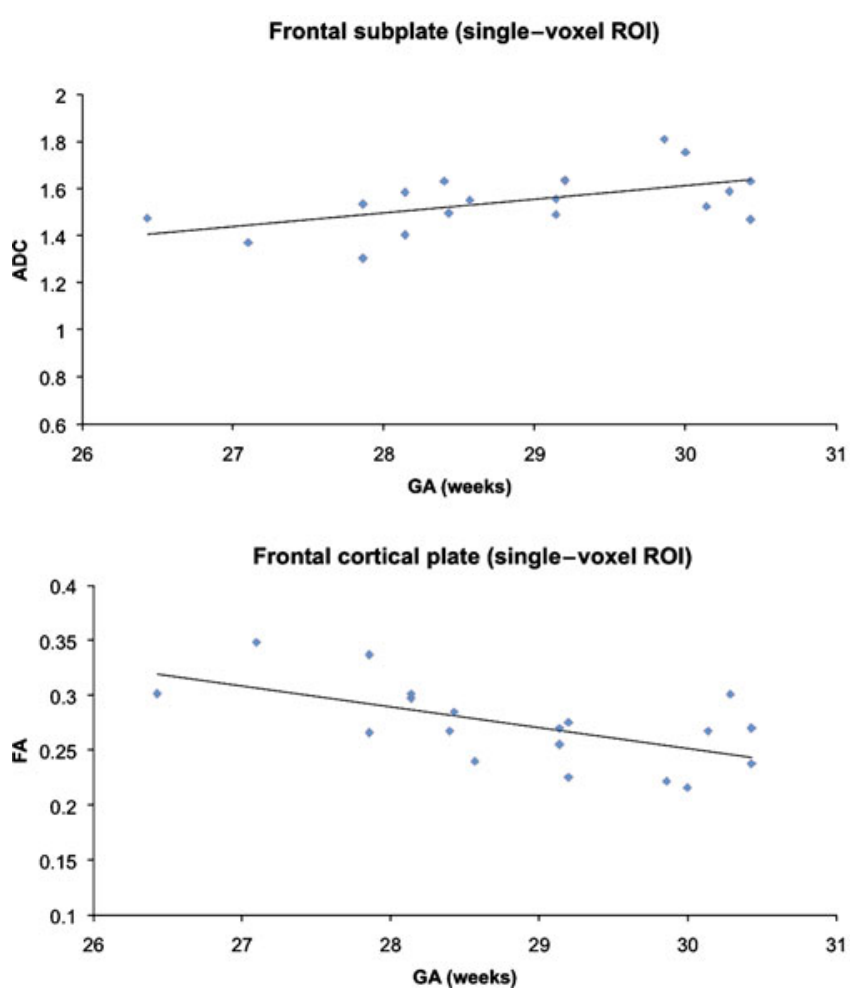

Fig. 3 FA and ADC of single-voxel ROIs cortical FA values have been reported. Neurons that form the cerebral CP migrate to their destinations along radial glial fibres [25]. It is thought that the observed increase in the cortical FA values during early GA ( $<27$ weeks) could be associated with this radial organization and with the radial orientation of the apical dendrites of pyramidal cells [26]. The decline in the cortical FA after 27 weeks probably reflects degradation in the radial organization. In time radial glial cells differentiate into astrocytes and gradually retract their ventricular and pial attachments [27]. In addition neocortical maturation will take place that results from neuronal growth, afferent ingrowth, dendrite elaboration and synapse formation [26]. These processes are an important basis of later functional connectivity. In agreement with others we have demonstrated a low cortical ADC. We
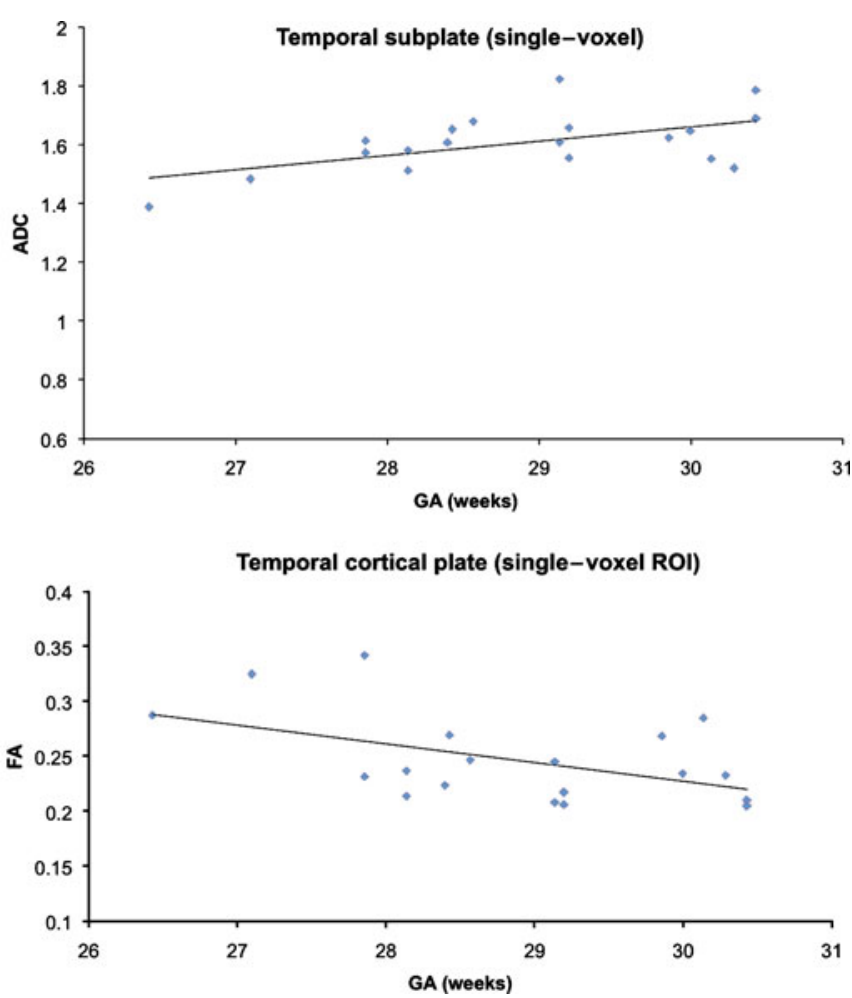


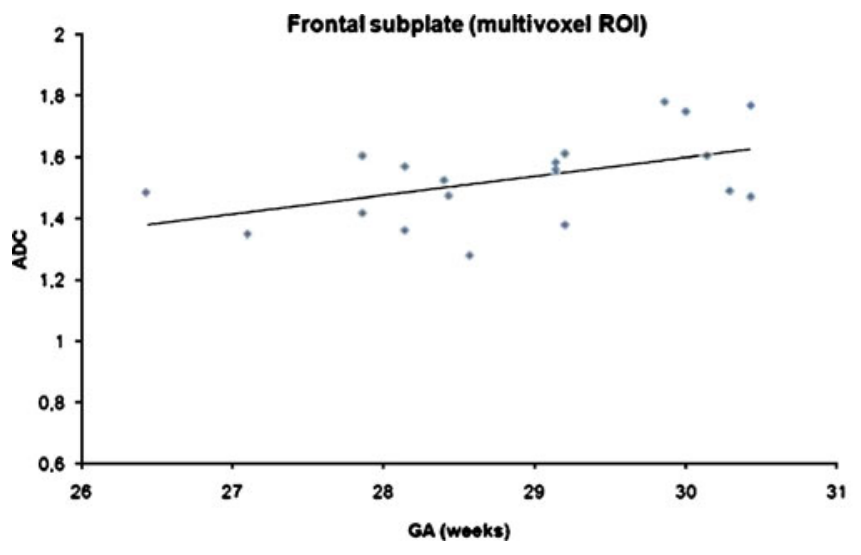

Fig. 4 FA and ADC of ROIs of standard 16-pixel size

did not find a significant correlation between GA and ADC values of $\mathrm{CP}$, which is probably due to the close age range of our infants. McKinstry et al. [18] did find an increase in $\mathrm{ADC}$ values of the $\mathrm{CP}$ until 32 weeks and a decrease hereafter. These divergent findings might reflect a difference of ROI location between the two studies. It is known that the rate of $\mathrm{CP}$ development varies depending on its location, which could also explain the differences found.

\section{$\mathrm{ADC}$ and $\mathrm{FA}$ values of subplate}

The subplate is a transient fetal cerebral zone, which serves as the 'waiting compartment' for growing cortical afferents [9]. The subplate is a laminar structure that consists of tissue with large extracellular spaces and an abundance of glycosaminoglycans [28]. It is most prominent around 22 weeks' gestation and disappears at around 32-34 weeks. In the ROIs corresponding with the subplate region we measured medium diffusivity and low anisotropy values. These findings seem to be consistent with earlier findings and might reflect the relatively lower cellularity of this region [14]. The finding of low FA values in the subplate region are consistent with the observation of more randomly scattered cells seen on histology, perhaps also due to the mixture of interdigitating radial and tangential fibres within this lamina $[14,29]$. We found an increase of ADC values in the region of the subplate between 26 weeks' and 31 weeks' gestation. These findings might be explained by the assumption that either a decrease in cellularity (associated with programmed cell death) in certain areas or the loss of interconnections of the brain could outweigh the decrease in overall water content during brain maturation [30-32].

Studying DTI parameters of the subplate of VLBW infants is challenging for many reasons. One of the challenges is to determine a practical ROI size, shape and location. We chose to examine the subplate in the temporal and frontal lobes because the subplate is clearly visible

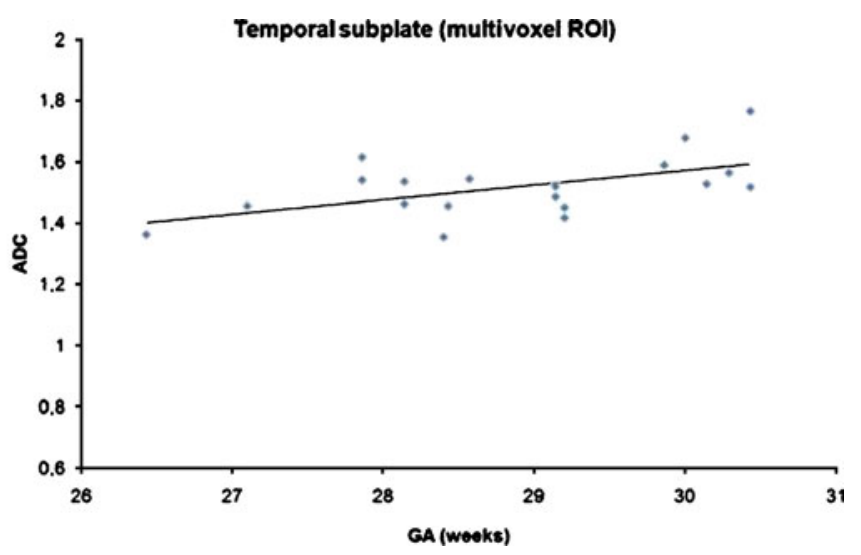

during the GAs studied and these regions are related to associative or 'executive' functions, which are increasingly recognized as being impaired in children born preterm.

A limitation of this study is that at 2 years of age it remains difficult to exclude global injury or interference with normal development with certainty. Although the developmental testing of premature infants at 2 years of age with the use of the Bayley scales of infant development provides an early opportunity to estimate future developmental capabilities, the correlation of developmental assessments with ultimate developmental achievement would be greater for assessments at 5 years of age or even later. Therefore, in future studies longer follow-up is necessary.

We compared two techniques to achieve better reproducibility. However both methods have limitations. In the single-voxel method, we used the best corresponding voxel from a row of voxels on a line crossing the subplate and CP. This technique is more prone to artefacts then a standard multivoxel ROI. The multivoxel ROI placed in the middle of a structure such as the subplate will be more prone to partial-volume effects. Because single-voxel ROI placement allows us to follow the changes of $\lambda 1, \lambda 2$ and $\lambda 3$ values seen in cross sections of the brain we found the single voxel method a useful way to study DTI parameters of the subplate and $\mathrm{CP}$. We found that the voxels with the lowest FA values correlated well with the voxels representing the middle of the $\mathrm{SP}$ region. However because of the limitations mentioned we recommend use of the multivoxel ROI method.

Using interobserver independent (semi-) automatic subplate segmentation might be the best way to cope with these limitations and will allow measurements of the complete subplate instead of a limited number of locations. However, the use of (semi-) automatic post processing algorithms does require specialized computing knowledge and will be less accessible for clinical purposes.

In this study we used a b-value of $1,000 \mathrm{~s} \mathrm{~mm}^{-2}$ to allow exact comparison between early and later scans of the study 
subjects made at different ages (including scans made at 6 weeks and 1 year). We realize a lower b-value for scanning the preterm brain would be optimal. The optimal b-value can be calculated as indicated by Jones et al [33]. The choice of DTI scan parameters (including b-value) have shown to affect both ADC and FA values. In a recent study we examined the effect of high b-values on FA and $\mathrm{ADC}$ in different neonatal brain regions. ADC values decreased with increasing b-value in all regions studied. In white matter, there was an approximately linear trend in the reduction of $\mathrm{ADC}$ with increasing b-value, whereas in the thalamus ADC declined more slowly at higher b-values. There was no consistent change in FA with increasing b-value in the neonatal brain [34].

Another serious challenge is the signal-to-noise ratio (SNR) and spatial resolution constraints due to the very low anisotropy of premyelinating white matter and the tiny size of white matter tracts in premature newborns. Using a custommade MR-compatible incubator with a high-sensitivity neonatal head coil that improved image quality and spatial resolution we were able to overcome these problems.

\section{Conclusion}

Secondary disturbances of cortical connectivity seem a logical anatomical substrate for some of the neurodevelopmental deficits seen in a large number of VLBW infants. Early diagnosis of subplate abnormalities is important for developing intervention strategies and for exploring different ways of perinatal brain repair.

Given the important role of the subplate in normal development of the $\mathrm{CP}$ and its selective vulnerability to hypoxicischaemic brain injury, a better understanding of normal subplate development is essential. Our study provides normal DTI reference values for evaluation of cortical brain maturation.

Open Access This article is distributed under the terms of the Creative Commons Attribution Noncommercial License which permits any noncommercial use, distribution, and reproduction in any medium, provided the original author(s) and source are credited.

\section{References}

1. Costeloe K, Hennessy E, Gibson AT et al (2000) The EPICure study: outcomes to discharge from hospital for infants born at the threshold of viability. Pediatrics 106:659-671

2. Piecuch RE, Leonard CH, Cooper BA et al (1997) Outcome of extremely low birth weight infants (500 to 999 grams) over a 12-year period. Pediatrics 100:633-639

3. Wood NS, Marlow N, Costeloe K et al (2000) Neurologic and developmental disability after extremely preterm birth. N Engl J Med 343:378-384

4. Hack M, Taylor HG, Klein N et al (2000) Functional limitations and special health care needs of 10- to 14-year-old children weighing less than 750 grams at birth. EIPCure Study Group. Pediatrics 106:554-560

5. Taylor HG, Klein N, Hack M (2000) School-age consequences of birth weight less than $750 \mathrm{~g}$ : a review and update. Dev Neuropsychol 17:289-321

6. Barnes PD (2001) Neuroimaging and the timing of fetal and neonatal brain injury. J Perinatol 21:44-60

7. Volpe JJ (1997) Brain injury in the premature infant. Neuropathology, clinical aspects, pathogenesis and prevention. Clin Perinatol 24:567587

8. Volpe JJ (2009) Brain injury in premature infants: a complex amalgam of destructive and developmental disturbances. Lancet Neurol 8:110-124

9. Kostovic I, Jovanov-Miloševic N (2008) Subplate zone of the human brain historical perspective and new concepts. Coll Antropol 32:38

10. Volpe JJ (1996) Subplate neurons-missing link in brain injury of the premature infant? Pediatrics 97:112-113

11. McQuillen PS, Sheldon RA, Shatz CJ et al (2003) Selective vulnerability of subplate neurons after early neonatal hypoxiaischemia. Neurosci 23:3308-3315

12. Ghosh A, Shatz CJ (1993) A role for subplate neurons in the patterning of connections from thalamus to neocortex. Development 117:1031-1047

13. Rados M, Judas M, Kostovic I (2006) In vitro MRI of brain development. Eur J Radiol 57:187-198

14. Maas LC, Mukherjee P, Carballido-Gamio J et al (2004) Early laminar organization of the human cerebrum demonstrated with diffusion tensor imaging in extremely premature infants. Neuroimage 22:1134-1140

15. Gupta RK, Hasan KM, Trivedi R et al (2005) Diffusion tensor imaging of the developing human cerebrum. J Neurosci 15:172178

16. Perkins L, Hughes E, Srinivasan L et al (2008) Exploring cortical subplate evolution using magnetic resonance imaging of the fetal brain. Dev Neurosci 30:211-220

17. Le Bihan D (2003) Looking into the functional architecture of the brain with diffusion MRI. Nat Rev Neurosci 4:469-480

18. McKinstry RC, Mathur A, Miller JH et al (2002) Radial organization of developing preterm human cerebral cortex revealed by non-invasive water diffusion anisotropy MRI. Cereb Cortex 12:1237-1243

19. Bayley N (1993) Bayley scores of infant development, 2nd edn. Psychological Corporation, San Antoni

20. Basser PJ, Pierpaoli C (1996) Microstructural and physiological features of tissues elucidated by quantitative-diffusion-tensor MRI. J Magn Reson B 111:209-219

21. Pierpaoli C, Basser PJ (1996) Toward a quantitative assessment of diffusion anisotropy. Magn Reson Med 36:893-906

22. Le Bihan D, Mangin JF, Poupon C et al (2001) Diffusion tensor imaging: concepts and applications. J Magn Reson Imaging 13:534 546

23. Dudink J, Kerr JL, Paterson K et al (2008) Connecting the developing preterm brain. Early Hum Dev 84:777-782

24. Trivedi R, Gupta RK, Husain N et al (2009) Region-specific maturation of cerebral cortex in human fetal brain: diffusion tensor imaging and histology. Neuroradiol 51:567-576

25. Rakic P (2003) Developmental and evolutionary adaptation of cortical radial glia. Cereb Cortex 13:541-549

26. Sidman RL, Rakic P (1973) Neural migration with special reference to developing human brain: a review. Brain Res 62:1-35

27. Volpe JJ (2008) Neurology of the newborn, 5th edn. Elsevier, Philadelphia, pp 1-1042

28. Kostovic I, Molliver ME (1974) A new interpretation of the laminar development of the cerebral cortex: synaptogenesis in different layers of neopallium in the human fetus. American 
association of anatomists. Eighty seventh annual session. Anat Rec 178:395

29. Mori S, Xue R, Zhang J et al (2002) High resolution anatomical study of human fetal brain development based on diffusion tensor imaging. Proc Int Soc Magn Reson Med 10:1305

30. Chan WY, Yew DT (1998) Apoptosis and Bcl-2 oncoprotein expression in the human fetal central nervous system. Anat Rec 252:165-175

31. Dobbing J, Sands J (1973) Quantitative growth and development of human brain. Arch Dis Child 48:757-767
32. Cannie M, De Keyzer F, Meersschaert J et al (2007) A diffusionweighted template for GA-related apparent diffusion coefficient values in the developing fetal brain. Ultrasound Obstet Gynecol 30:318-324

33. Jones DK, Horsfield MA, Simmons A (1999) Optimal strategies for measuring diffusion in anisotropic systems by magnetic resonance imaging. Magn Reson Med 42:515-525

34. Dudink J, Larkman DJ, Kappellou O et al (2008) High b-value diffusion tensor imaging of the neonatal brain at $3 \mathrm{~T}$. AJNR 29:1966-1972 